\title{
A sucção não nutritiva do recém-nascido prematuro como uma tecnologia de enfermagem
}

\author{
The non-nutritive sucking of premature newborn as a nursing technology \\ La succión no nutritiva de los recién nacidos prematuros como una tecnología de enfermería
}

\section{Joice Cristina Pereira Antunes', Maria Aparecida de Luca Nascimento"}

' Fundação Oswaldo Cruz, Instituto Nacional Fernandes Figueira, Departamento de Enfermagem Neonatal. Rio de Janeiro-RJ, Brasil. "Universidade Federal do Estado do Rio de Janeiro, Centro de Ciências Biológicas e da Saúde, Escola de Enfermagem Alfredo Pinto. Rio de Janeiro-RJ, Brasil.

\author{
Submissão: 16-04-2012 Aprovação: 22-07-2013
}

\section{RESUMO}

Estudo experimental com abordagem quantitativa, cujo objetivo foi demonstrar que a sucção não nutritiva é efetiva no manejo da dor durante a instalação, pela equipe de enfermagem, do CPAP nasal em recém-nascidos prematuros; e demonstrar que o uso da sucção não nutritiva, concomitantemente à instalação do CPAP nasal, pode ser considerado uma tecnologia de enfermagem. A população alvo foi constituída por 20 recém-nascidos prematuros, submetidos à instalação ou reinstalação do referido artefato, totalizando 30 procedimentos. Os recém-nascidos foram distribuídos, aleatoriamente, em dois grupos, controle e experimental, em que a sucção não nutritiva foi oferecida, o mesmo não acontecendo com o grupo controle. As reações de dor foram mensuradas pela escala de NIPS. Em 100\% dos procedimentos concomitantes à sucção não nutritiva, os recém-nascidos não sentiram dor; $100 \%$ dos recém-nascidos demonstraram dor quando não era oferecida a referida sucção. Conclui-se que o procedimento pode ser classificado como uma tecnologia do cuidado de enfermagem.

Descritores: Enfermagem Neonatal; Cuidados de Enfermagem; Tecnologia; Sucção não Nutritiva.

This is an experimental study with a quantitative approach, whose goal was to demonstrate that non-nutritive sucking is effective in pain management during installation, by the nursing staff, of nasal CPAP in preterm infants; and to demonstrate that the use of non-nutritive sucking, concomitantly with the installation of nasal CPAP can be considered a nursing technology. The target population consisted of 20 preterm infants undergoing installation or reinstallation of this artifact, totaling 30 procedures. The newborns were divided randomly into two groups, control and experimental, in which non-nutritive sucking was offered, the same do not happening with the control group. The reactions of pain were measured by the scale of NIPS. In $100 \%$ of the procedures that occurred concomitant with non-nutritive sucking, newborns did not feel pain; and 100\% of the newborns showed pain when such suction was not offered. We conclude that the procedure can be classified as a technology of nursing care.

Key words: Neonatal Nursing; Nursing Care; Technology; Non Nutritive Sucking.

\section{RESUMEN}

Estudio experimental con un enfoque cuantitativo, cuyo objetivo fue demostrar que la succión no nutritiva es eficaz en el tratamiento del dolor durante la instalación, por personal de enfermería, de la CPAP nasal en bebés prematuros; y demostrar que el uso de la succión no nutritiva, concomitantemente a la instalación de la CPAP nasal, puede ser considerada una tecnología de enfermería. La población-objetivo consistió en 20 recién nacidos prematuros sometidos a la instalación o reinstalación de este artefacto, en un total de 30 procedimientos. Los recién nacidos fueron divididos aleatoriamente en dos grupos, control y experimental, en el que se ofrece la succión no nutritiva, lo mismo no ocurriendo con el grupo control. Las reacciones de dolor se midieron por la escala de NIPS. En 100 \% de los procedimientos concomitantes con succión no nutritiva, los recién nacidos no sentirán dolor; y 100 \% de los recién nacidos mostró dolor cuando no se le ofreció la succión. Llegamos a la conclusión de que este procedimiento puede ser clasificado como una tecnología de cuidado de enfermería.

Palabras clave: Enfermería Neonatal; Atención de Enfermería; Tecnología; Succión No Nutritiva. 


\section{INTRODUÇÃO}

Até o final do século XIX, as elevadas taxas de mortalidade entre os recém-nascidos de baixo peso ainda eram inevitáveis. Após a segunda metade do século $\mathrm{XX}$, o avanço tecnológico ligado ao cuidado perinatal, foi responsável por um decréscimo significativo na mortalidade neonatal, particularmente do recém-nascido prematuro (RNPT) ${ }^{(1)}$.

Desse modo, a sobrevida neonatal, está diretamente ligada ao avanço das tecnologias, entre elas, aquelas relacionadas à assistência ventilatória, que oferece suporte à principal vulnerabilidade desta clientela, que é a imaturidade pulmonar, a partir da qual vários dispositivos foram construídos com o objetivo, não somente de simular os movimentos respiratórios, como também, de melhorar a função pulmonar, e consequentemente, a redução de apneias.

Atualmente, entre os dispositivos mais utilizados na assistência ventilatória do RNPT, disponíveis nas unidades de tratamento intensivo neonatal (UTIN) estão, o oxihood, a pressão positiva contínua nas vias aéreas e a ventilação mecânica.

O oxihood é um dispositivo em forma de capacete que administra oxigênio e ar comprimido, umidificados e aquecidos, sendo indicado para os recém-nascidos (RNs) que respiram espontaneamente, mas que precisam de uma concentração de oxigênio inferior a $60 \%$. Nos casos dos RNs que necessitam de oxigênio em maior concentração, e que têm um desconforto respiratório associado a essa necessidade, a utilização da pressão positiva contínua nas vias aéreas é indicada. Porém, se o problema do RN estiver relacionado à inabilidade dos seus pulmões em manter uma ventilação adequada, será indicada a ventilação mecânica através da intubação endotraqueal ${ }^{(2)}$.

Entre os dispositivos tecnológicos acima mencionados, destacamos o CPAP nasal (Continuous Positive Airway Pressure), cuja tradução quer dizer pressão positiva contínua nas vias aéreas, sendo frequentemente utilizado no tratamento de RNs com doenças que cursam com a capacidade residual funcional diminuída, tais como a doença da membrana hialina, a taquipneia transitória do RN e a displasia broncopulmonar.

A utilização da ventilação de pressão positiva contínua nas vias aéreas (CPAP) via endotraqueal como tratamento da angústia respiratória da prematuridade, foi satisfatoriamente utilizada em $1973^{(3)}$, ressaltando-se que essa modalidade ventilatória proporciona efeitos fisiológicos nos recém-nascidos, sobretudo nos prematuros, tendo em vista consistir na manutenção de uma pressão supra-atmosférica durante a expiração em um paciente que respira espontaneamente, favorecendo ao recrutamento dos alvéolos, além de permitir que os alvéolos colapsados sejam inflados.

Porém, a melhoria na oxigenação diminui a vasoconstrição no leito vascular, contribuindo para a queda da resistência vascular pulmonar, permitindo um aumento no fluxo pulmonar com consequente aumento da pressão parcial de oxigênio $\left(\mathrm{paO}_{2}\right)$. Além de favorecer não somente a redução de apneias obstrutivas, já que estabiliza o diafragma, o CPAP nasal promove um ritmo respiratório regular pela estabilização da parede torácica.
A modalidade ventilatória em foco ainda contribui para a liberação do surfactante, substância armazenada nos alvéolos, que permite o aumento da complacência pulmonar, sendo imprescindível para que os alvéolos se mantenham adequadamente inflados e com menor gasto energético do organismo ${ }^{(2)}$.

O CPAP nasal é indicado essencialmente em RNPT com respiração espontânea, portadores da doença de Membrana Hialina (deficiência de surfactante) branda ou moderada; na apneia da prematuridade e nos casos de retirada do tubo orotraqueal, ao término da sua indicação clínica ${ }^{(4)}$.

Este estudo tem como foco, não só, as tecnologias que são utilizadas diuturnamente pela equipe de enfermagem no cuidado ao RNPT, como também, a proposta de considerar como tecnologias, os procedimentos técnicos de enfermagem que são utilizados durante essa mesma assistência.

Neste sentido, podemos dizer que as medidas não farmacológicas utilizadas para prevenir ou reduzir a intensidade de um processo doloroso deve ser considerada como tecnologia. Desta forma, ao utilizar a tecnologia da sucção não nutritiva durante a instalação do artefato CPAP nasal, a enfermagem neonatal estará se apropriando de uma tecnologia do cuidado que proporcionará o alívio da dor no recém-nascido prematuro, e consequentemente, a melhora de sua sobrevida.

É importante ressaltar que o cuidado de enfermagem está intimamente interligado à tecnologia, tendo em vista que os profissionais de enfermagem estão comprometidos com princípios, leis e teorias e a tecnologia representa esse conhecimento científico e sua própria transformação ${ }^{(5)}$.

Face ao exposto, cumpre citar que o uso das tecnologias na área da saúde, necessita ser expandido, não significando apenas a incorporação de equipamentos no cuidado ${ }^{(6)}$.

Considerando que a observância dos aspectos científicos que permeiam o cuidado de enfermagem permitem distingui -la como uma profissão cujo menor cuidado deve ser provido da maior atenção, tendo em vista a dignidade da criança que está sendo alvo do nosso cuidado(7), e que, ao praticá-lo os profissionais de enfermagem tem a possibilidade, através da observação, de detectar sinais impressos no corpo, que indicam sintomas muitas vezes não referidos ${ }^{(8)}$, este estudo teve como objetivos: demonstrar que o uso da sucção não nutritiva, pela equipe de enfermagem, é efetiva no manejo da dor durante a instalação do CPAP nasal em recém-nascidos prematuros (RNPT); demonstrar que o uso da sucção não nutritiva, concomitantemente à instalação do CPAP nasal, pode ser considerado uma tecnologia de enfermagem.

\section{METODOLOGIA}

Estudo experimental com abordagem quantitativa, que tem, entre outras características, a manipulação de um determinado fator, (que para efeito desse estudo, será a sucção não nutritiva), e que, a critério do pesquisador, gerará um determinado resultado em um outro fator (manifestação de dor do RNPT durante a instalação do CPAP nasal). Este outro fator é a chamada variável dependente, que sofrerá, ou não, um efeito da manipulação da variável independente, sendo medido o seu resultado para determinar a validade do experimento ${ }^{(9)}$. 
Quadro 1 - Número de instalação e reinstalação do CPAP nasal a que cada RNPT foi submetido.

\begin{tabular}{|c|c|c|c|c|c|c|c|c|c|c|c|c|c|c|c|c|c|c|c|c|}
\hline RNPT & A & B & C & D & $\mathbf{E}$ & $\mathbf{F}$ & $\mathbf{G}$ & $\mathbf{H}$ & $\mathbf{I}$ & $\mathbf{J}$ & $\mathbf{K}$ & $\mathbf{L}$ & $\mathbf{M}$ & $\mathbf{N}$ & $\mathbf{O}$ & $\mathbf{P}$ & $\mathbf{Q}$ & $\mathbf{R}$ & $\mathbf{S}$ & $\mathbf{T}$ \\
\hline $\begin{array}{c}\text { No de } \\
\text { (re)instalação } \\
\text { do CPAPn }\end{array}$ & 1 & 1 & 1 & 1 & 1 & 1 & 1 & 1 & $\mathbf{5}$ & 1 & 1 & $\mathbf{5}$ & $\mathbf{2}$ & $\mathbf{2}$ & 1 & 1 & 1 & 1 & 1 & 1 \\
\hline
\end{tabular}

A população alvo foi composta por 20 RNPT, durante a instalação ou reinstalação do CPAP nasal, num total de 30 procedimentos, que foram distribuídos em grupo controle (sob sucção não nutritiva), ou experimental (sem sucção não nutritiva).

A cada procedimento, a distribuição aleatória era feita, sendo essa randomização realizada após o lançamento de uma moeda, a partir da face apresentada.

Desta forma, foram observados 30 procedimentos de instalação do CPAP nasal, realizados em 20 RNPTs, com as mesmas características de elegibilidade. O quadro 1 mostra o número de instalação e reinstalação do CPAP nasal a que cada RNPT foi submetido.

De acordo com o quadro acima, podemos perceber que os prematuros $\mathbf{I}, \mathbf{L}, \mathbf{M}$ e $\mathbf{N}$, necessitaram reinstalar o CPAP nasal pela piora do seu desconforto respiratório, sendo que os RNPTs I e $\mathbf{L}$ foram submetidos cinco vezes a este estímulo doloroso e estressante, enquanto que os RNPTs $\mathbf{M}$ e $\mathbf{N}$ foram submetidos a ele por duas vezes.

A observação sistemática e não participativa foi feita a partir de variáveis comportamentais (expressão facial, choro, movimentação de braços e pernas) e fisiológicas (padrão respiratório) que compõem a escala de NIPS, e que foram apresentadas pelo RNPT durante a instalação do CPAP nasal.

Segundo a escala em referência, ao final da sua aplicação é realizado o somatório dos pontos que podem estar compreendidos entre 0 e 7 , sendo que o score igual ou superior a 4 é indicativo de dor.

A realização da sucção não nutritiva foi estabelecida através da introdução do dedo mínimo enluvado na cavidade oral do recém-nascido prematuro, concomitantemente à instalação do CPAP nasal, não sem antes retirar-se o excesso de talco com água destilada, que, porventura, pudesse causar algum malefício para RNPT.
A observação e a instalação do CPAP nasal foram realizadas pelas residentes de enfermagem em neonatologia no segundo ano de residência (totalizando três enfermeiras). A fim de minimizar a ameaça à validade interna do estudo através do fator instrumentação, as residentes foram orientadas e treinadas com relação aos seguintes aspectos: modo de aplicação da escala de NIPS, maneira de proceder à observação, ao preenchimento do instrumento de coleta de dados e à forma de instalar o CPAP nasal com a utilização ou não da sucção não nutritiva.

No que tange à instalação do CPAP nasal, o procedimento seguiu os seguintes passos:

$1^{\circ}$ passo: Organização do material necessário para a instalação do CPAP nasal

$\mathbf{2}^{\mathbf{0}}$ passo: A lavagem das mãos tem que ser precedida ao cuidado com o recém-nascido, principalmente os RNPTs, já que apresentam maior deficiência da resposta imunológica, sendo mais propensos à infecção.

Deve ser realizada até os antebraços, com água e sabão, durante 15 segundos; sendo necessário retirar todos os acessórios que estejam nas mãos e pulsos, tais como: relógio, pulseiras e anéis ${ }^{(10)}$.

$3^{\circ}$ passo: Separa-se a pronga que se adequa à narina do recém-nascido (que deveria ser de acordo com o diâmetro da narina do RN, mas é de acordo com o seu peso); a touca necessária para estabilizar as traqueias na cabeça do bebê; as traqueias de hudson (são duas) e, o hidrocolóide cortado no formato da narina a fim de proteger a mucosa e atenuar o escape de ar nas narinas.

$4^{\mathbf{0}}$ passo: Posicionar adequadamente o recém-nascido prematuro, mantendo os membros fletidos e aproximados, o máximo possível, à linha média do corpo, com a cabeça elevada aproximadamente 30 graus, entendendo que o posicionamento adequado favorece a auto-organização, minimiza

Escala de NIPS - Neonatal Infant Pain Scale / Escala de Dor Neonatal.

\begin{tabular}{|l|c|c|c|}
\hline \multicolumn{1}{|c|}{ Indicador } & 0 ponto & 1 ponto & 2 pontos \\
\hline 1) Expressão facial & Relaxada & Contraída & \\
\hline 2) Choro & Ausente & "Resmungos" & Vigoroso \\
\hline 3) Respiração & Relaxada & Diferente da basal & - \\
\hline 4) Braços & Relaxados & Fletidos ou estendidos & - \\
\hline 5) Pernas & Relaxadas & Fletidos ou estendidos & - \\
\hline 6) Estado de Alerta & Dormindo ou acordado calmo & Desconfortável & - \\
\hline
\end{tabular}

Fonte: Guinsburg, 1999. 
a situação do estresse e ajuda a manter o prematuro estável durante a execução deste procedimento, causador de desagradáveis estímulos ${ }^{(11)}$.

$5^{\circ}$ passo: Colocar a touca no recém-nascido prematuro; acoplar as duas traqueias às laterais da pronga, posicionando-a, cuidadosamente, na narina dele, onde já está fixado o hidrocolóide.

Vale ressaltar que este procedimento deverá ser realizado por dois profissionais, pois enquanto um profissional instala a pronga nasal, o outro estará oferecendo oxigênio inalatório (até 3L/ min) enquanto a pronga nasal é, cuidadosamente, introduzida, o mínimo possível, para gerar a pressão positiva. Paralelamente à introdução da pronga, faz-se necessário que as traqueias em sua porção distal sejam acopladas ao respirador e, na porção proximal, fixadas paralelamente à touca do recém-nascido com fitas adesivas ou de esparadrapo.

É válido enfatizar que a mobilidade excessiva da pronga em relação às narinas poderá ocasionar trauma na epiderme e/ou mucosa, e, contribuir para flutuações na oferta de oxigênio e pressão positiva ao RNPT. Desta forma, este procedimento somente finaliza quando ocorre a perfeita adequação do RNPT à modalidade ventilatória, sendo talvez o momento mais delicado de todo o procedimento, pois requer paciência e um cuidado diferenciado e individualizado.

O mesmo recém-nascido prematuro foi submetido ao grupo controle e ao grupo experimental em momentos diferentes, uma vez que, se houve necessidade de retornar para o CPAP nasal, um novo sorteio foi realizado para determinar a utilização ou não da sucção não nutritiva. As observações foram restringidas ao momento de instalação do dispositivo nasal.

O projeto do qual derivou este estudo, foi aprovado pelo Comitê de Ética em Pesquisa com Seres Humanos do Instituto Fernandes Figueira (CEP-IFF), através do protocolo nº 0044/08 de $27 / 03 / 2009$.

\section{RESULTADOS}

A observação do procedimento de instalação ou reinstalação do CPAP nasal foi realizada nos grupos experimental e controle, sendo que 11 foram feitas no grupo experimental e 19 no grupo controle, totalizando 30 observações.
Considerando a pontuação da escala de NIPS, conforme o quadro demonstrativo 2, a observação do procedimento em foco, demonstrou que o score prevalente no grupo controle foi 6 em 47,4\% (9) das observações, seguido pelo score 7 com $31,6 \%$ (6) e 5 em $21 \%$ (4). Em contrapartida, no grupo experimental o score máximo alcançado foi 3 em $9 \%$ (1), sendo o score igual a 1 prevalente em $82 \%$ (9) das observações.

Estes resultados indicam que, ao instalar o CPAP nasal, $100 \%$ dos RNPTs sentem dor. No entanto, quando lhes é oferecida a sucção não nutritiva, eles reagem ao estímulo sem atingir uma pontuação indicativa de dor.

Podemos perceber que, no grupo experimental, a pontuação 1 foi prevalente em $82 \%$ das observações. Este fato ocorre porque o corpo humano é projetado física e mentalmente para reagir a qualquer estímulo externo ou interno, e uma mudança na sua harmonia pode levar a respostas comportamentais ou fisiológicas que culmina em um estado de conforto ou desconforto $^{(11)}$

Neste caso, quando se trata do estímulo em foco, a experiência mostrou que a instalação ou reinstalação do CPAP nasal associada a uma intervenção não farmacológica (sucção não nutritiva), levou a respostas comportamentais ou fisiológicas que culminaram em um estado de conforto, quando ocorre uma completa harmonia do RNPT.

Diversos estudos randomizados têm evidenciado que a sucção não nutritiva é um fator minimizador da dor do neonato durante a realização de procedimentos dolorosos ${ }^{(12-13)}$, corroborado pelo resultado de estudo que evidenciou a preocupação da equipe de enfermagem com o bem-estar dos RNPT internados em uma UTIN ${ }^{(14)}$.

\section{CONCLUSÃO}

Aliviar a dor dos RNPTs tem sido um grande desafio para a equipe de enfermagem, tendo em vista, não só a vulnerabilidade dessa clientela, como também, a sensibilidade do observador, em detectar através da observação apurada, sinais indicativos de sentimentos impressos no seu corpo diminuto.

Contudo, ao observarmos as necessidades que são inerentes ao RNPT, podemos inferir que não são poucos os procedimentos tecnológicos aos quais eles estão expostos, uma vez

Quadro 2 - Pontuação da escala de NIPS nos RNPTs do grupo experimental e do grupo controle.

\begin{tabular}{|c|c|c|c|c|}
\hline \multirow{2}{*}{$\begin{array}{c}\text { PONTUAÇÃO DA } \\
\text { ESCALA DE NIPS }\end{array}$} & \multicolumn{2}{|c|}{$\begin{array}{c}\text { GRUPO EXPERIMENTAL } \\
\text { GRUPO CONTROLE } \\
\text { (com sucção não nutritiva) }\end{array}$} \\
\cline { 2 - 5 } & N & \% & N sucção não nutritiva) \\
\hline 0 & 1 & 9 & 0 & 0 \\
1 & 9 & 82 & 0 & 0 \\
3 & 0 & 0 & 0 & 0 \\
4 & 1 & 9 & 0 & 0 \\
6 & 0 & 0 & 0 & 0 \\
7 & 0 & 0 & 4 & 0 \\
\end{tabular}


que, à medida que a ciência avança, novos recursos são disponibilizados no vasto mercado da assistência à saúde, quase sempre, demandando uma sensação dolorosa durante a sua utilização.

Esse estudo demonstrou que a instalação do CPAP nasal é um procedimento doloroso, e que a sucção não nutritiva é eficaz no manejo dessa sensação, durante a sua instalação.
Face ao exposto, cumpre citar que a oferta da sucção não nutritiva pela equipe de enfermagem, durante a instalação do CPAP nasal, deve ser considerada como uma tecnologia de enfermagem, tendo em vista que o cuidado de enfermagem e a tecnologia estão interligados, especialmente em situações críticas da vida, onde o adequado desenvolvimento neurológico de um prematuro é o maior objetivo da equipe neonatal.

\section{REFERÊNCIAS}

1. Ramos HAC, Cuman RKN. Fatores de risco para prematuridade: pesquisa documental. Esc Anna Nery Rev Enferm 2009;13(2):297-304.

2. Tamez RN, Silva MJP. Enfermagem na UTI Neonatal. 4. ed. Rio de Janeiro: Guanabara Koogan; 2009.

3. Muhlhausen GM. Uso actual de présion positiva continua en la via aérea (CPAP)em recién nacidos. Pediatría (Santiago de Chile)2004:1(1);141-4.

4. Macdonald MG, Mullett MD, Seshia MMK. Avery/Neonatologia: fisiopatologia e tratamento do recém-nascido. 6 ed. Rio de Janeiro: Guanabara Koogan; 2007. p.1634.

5. Rocha PK, Prado ML, Wal ML, Carraro TE. Cuidado e tecnologia: aproximações através do modelo de cuidado. Rev Bras Enferm 2008;61(1):113-6.

6. Schwonke CRGB, Lunardi Filho WD, Lunardi VL, Santos SSC, Barlem ELD. Perspectivas filosóficas do uso da tecnologia no cuidado de enfermagem em terapia intensiva. Rev Bras Enferm 2010;64(1):189-92.

7. Panunto MR, Guirardello EB. Carga de trabalho de enfermagem em unidade de terapia intensiva de um hospital de ensino. Acta Paul Enferm 2012;(25):1.

8. Araujo MC, Nascimento MAL, Christoffel MM, Antunes
JCP, Gomes AVO. Aspiração traqueal e dor: reações do recém-nascido pré-termo durante o cuidado. Cienc Cuid Saúde 2010;9(2):255-261.

9. Polit DF, Beck CT, Hungler BP. Fundamentos da pesquisa em enfermagem: avaliação de evidência para a prática da enfermagem. 7. ed. [S.I.]: Artmed; 2011.

10. Angência Nacional de Vigilância Sanitária. Indicadores nacionais de infecções relacionadas à assistência à saúde. Brasília, DF: ANVISA; 2010.

11. Antunes JCPA, Nascimento MAL, Gomes AVO, Campos MA, Christoffel MM. Tecnologia coadjuvante no tratamento do recém-nascido prematuro (cuidados de enfermagem no uso do CPAP nasal). Enferm Glob 2010;(20):1-11.

12. Farias LM, Rêgo RMV, Lima FET, Araújo TL, Cardoso MVLML, Souza AMA. Cuidados de enfermagem no alívio da dor de recém-nascido: Revisão integrativa. Rev RENE 2011;12(4):866-74.

13. Aquino FM, Christoffel MM. Dor Neonatal: Medidas não farmacológicas utilizadas pela equipe de enfermagem. Rev RENE 2010;11:169-177.

14. Perencini CC, Ribeiro CA. Tocando o prematuro: significado para auxiliares e técnicas de enfermagem. Rev Bras Enferm 2011;64(5):817-23. 